\title{
On volumes determined by subsets of Euclidean space
}

\author{
Allan Greenleaf, Alex Iosevich and Mihalis Mourgoglou
}

\begin{abstract}
Given $E \subset \mathbb{R}^{d}$, define the volume set of $E, \mathcal{V}(E)=\left\{\operatorname{det}\left(x^{1}, x^{2}, \ldots x^{d}\right): x^{j} \in E\right\}$. In $\mathbb{R}^{3}$, we prove that $\mathcal{V}(E)$ has positive Lebesgue measure if either the Hausdorff dimension of $E \subset \mathbb{R}^{3}$ is greater than $\frac{13}{5}$, or $E$ is a product set of the form $E=B_{1} \times B_{2} \times B_{3}$ with $B_{j} \subset \mathbb{R}, \operatorname{dim}_{\mathcal{H}}\left(B_{j}\right)>\frac{2}{3}, j=1,2,3$. We show that the same conclusion holds for $\mathcal{V}(E)$ of Salem subsets $E \subset \mathbb{R}^{d}$ with $\operatorname{dim}_{\mathcal{H}}(E)>d-1$, and give applications to discrete combinatorial geometry.
\end{abstract}

\section{Introduction}

A large class of Erdős type problems in geometric combinatorics ask whether a large set of points in Euclidean space determines a suitable large sets of geometric relations, configurations or objects. For example, the classical Erdős distance problem asks whether $N$ points in $\mathbb{R}^{d}, d \geq 2$, determines $\gtrsim N^{\frac{2}{d}}$ distinct distances. See, for example $[1,13,15,16,17]$ and the references there for thorough descriptions of these types of problems and recent results. (Here, and throughout, $X \lesssim Y$, if the controlling parameter is $N$, means that for every $\epsilon>0$ there exists $C_{\epsilon}>0$ such that $X \leq C_{\epsilon} N^{\epsilon} Y$, while $X \lesssim Y$ means $X \leq C Y$ with $C$ independent of $N$.)

Continuous variants of Erdős type geometric problems have also received much attention in recent decades. Perhaps the best known of these is the Falconer distance problem [7], which asks whether the one-dimensional Lebesgue measure of the distance set $\{|x-y|: x, y \in E\}$ is positive, provided that the Hausdorff dimension, $\operatorname{dim}_{\mathcal{H}}(E)$, of $E \subset \mathbb{R}^{d}, d \geq 2$, is greater than $\frac{d}{2}$. See $[\mathbf{1 9}, 4]$ for the best currently known results on this problem. Also see [5] for the closely related problem of finite point configurations.

In this paper we study the sets of volumes determined by sets $E \subset \mathbb{R}^{d}$. Given $d$ vectors $x^{1}, x^{2}, \ldots x^{d}$ in $\mathbb{R}^{d}, d \geq 2$, let $\operatorname{det}\left(x^{1}, \ldots, x^{d}\right)$ denote the determinant of the matrix whose $j$ th column is $x^{j}$. For $E \subset[0,1]^{d}$, define the volume set of $E$,

$$
\mathcal{V}(E):=\left\{\operatorname{det}\left(x^{1}, x^{2}, \ldots, x^{d}\right) \in \mathbb{R}: x^{j} \in E\right\} .
$$

A problem is to find the optimal theshhold $s_{\mathcal{V}}(d)$ such that if the Hausdorff dimension of $E \subset[0,1]^{d}$ is greater than $s_{\mathcal{V}}(d)$, then the Lebesgue measure of $\mathcal{V}(E)$, denoted by $\mathcal{L}^{1}(\mathcal{V}(E))$, is positive. Letting $E$ be a $(d-1)$-dimensional hyperplane shows that one must take $s \mathcal{V}(d) \geq d-1$.

The first two authors authors were supported by NSF grants DMS-0853892 and DMS-1045404. The third author holds a Sophie Germain International post-doctoral scholarship at Fondation de Mathématiques Jacques Hadamard (FMJH) and would like to thank the faculty and staff of the Universite Paris-Sud 11, Orsay for their hospitality. 
A result due to Erdog̃an, Hart and the second author [6] shows that $s_{\mathcal{V}}(2) \leq \frac{3}{2}$. More generally, they prove the following result.

Theorem 1.1. [6] For $d \geq 2$, let $E, F \subset[0,1]^{d}$ and $Q$ be a non-degenerate bilinear form on $\mathbb{R}^{d}$. Suppose that $\operatorname{dim}_{\mathcal{H}}(E)+\operatorname{dim}_{\mathcal{H}}(F)>d+1$. Then $\mathcal{L}^{1}(\{Q(x, y): x \in E, y \in F\})>0$.

Thus, if $E \subset \mathbb{R}^{2}$ with $\operatorname{dim}_{\mathcal{H}}(E)>\frac{3}{2}$, then $\mathcal{L}^{1}(\mathcal{V}(E))>0$.

Our four main results are the following.

Theorem 1.2. Let $E \subset[0,1]^{3}$, with $\operatorname{dim}_{\mathcal{H}}(E)>\frac{13}{5}$. Then $\mathcal{L}^{1}(\mathcal{V}(E))>0$.

In view of Thm. 1.1 above, Thm. 1.2 may be reduced to studying the Hausdorff dimension of the set of $(d-1)$-vectors of $E$,

$$
\Lambda(E):=\left\{*\left(x^{1} \wedge x^{2} \wedge \cdots \wedge x^{d-1}\right): x^{j} \in E, 1 \leq j \leq d-1\right\},
$$

where $*$ is the Hodge star operator, $*: \Lambda^{d-1} \mathbb{R}^{d} \longrightarrow \mathbb{R}^{d}$. Since

$$
\operatorname{det}\left(x^{1}, \ldots, x^{d}\right)= \pm x^{d} \cdot *\left(x^{1} \wedge x^{2} \wedge \cdots \wedge x^{d-1}\right),
$$

establishing Thm. 1.2 reduces to proving the following result.

Theorem 1.3. Let $E \subset[0,1]^{3}$ and suppose that $\operatorname{dim}_{\mathcal{H}}(E)>\frac{13}{5}$. Then

$$
\operatorname{dim}_{\mathcal{H}}(E)+\operatorname{dim}_{\mathcal{H}}(\Lambda(E))>4 .
$$

If one could strengthen (1.3) to say that the left hand side is greater than 3 , then Thm. 1.2 would be improved to the $\operatorname{dim}_{\mathcal{H}}(E)>2$. This would be optimal because, as noted above, the conclusion of Thm. 1.2 does not in general hold if $\operatorname{dim}_{\mathcal{H}}(E) \leq d-1$.

Remark 1.4. In the context of three dimensional vector spaces over finite fields, the analogue of Theorem 1.2 is completely resolved, with a sharp exponent using sum-product technology; see [2]. However, sum-product issues are generally believed to be more difficult in the continuous setting.

It is possible to obtain a better exponent than in Thm. 1.3, and thus Thm. 1.2, if the set $E$ has a special form or satisfies Fourier decay conditions. We first consider the situation where the set under consideration is a Cartesian product of subsets of the real line.

Theorem 1.5. Suppose that $E=B_{1} \times B_{2} \times B_{3}$, where $B_{j} \subset[0,1] \operatorname{satisfy}_{\operatorname{dim}}\left(B_{j}\right)>\frac{2}{3}, j=1,2,3$. Then $\mathcal{L}^{1}(\mathcal{V}(E))>0$.

Note that if each $B_{j}$ is Ahlfors-David regular (see e.g. [14]), the assumption that the Hausdorff dimension of each $B_{j}$ is greater than $\frac{2}{3}$ is equivalent to the assumption that the Hausdorff dimension of $E=B_{1} \times B_{2} \times B_{3}$ is greater than 2. See, e.g., [8, 14].

Alternatively, one can also consider the situation in higher dimensions, when set $E \subset \mathbb{R}^{d}, d \geq 2$, supports a measure that has good Fourier decay properties. Recall that $E \subset[0,1]^{d}, d \geq 2$, is Salem if there exists a probability measure $\mu$ supported on $E$ such that

$$
|\widehat{\mu}(\xi)| \lesssim|\xi|^{-\frac{s}{2}}, \quad|\xi| \longrightarrow \infty
$$

where $s$ denotes the Hausdorff dimension of $E$ and

$$
\widehat{\mu}(\xi)=\int e^{-2 \pi i x \cdot \xi} d \mu(x)
$$

is the Fourier transform of $\mu$. We prove 
Theorem 1.6. Suppose $E \subset \mathbb{R}^{d}$ is Salem and $s:=\operatorname{dim}_{\mathcal{H}}(E)>d-1$. Then $\mathcal{L}^{1}(\mathcal{V}(E))>0$.

We note that while one cannot do better than the exponent $d-1$, as the example of a hyperplane shows, it is possible that this might not be the case for Salem sets. It is conceivable that the conclusion of Theorem 1.6 holds if the Hausdorff dimension of $E$ is merely greater than one.

\section{Proof of Theorem 1.6}

Let $\psi$ be a smooth cut-off function on $\mathbb{R}^{d}, \equiv 1$ in the unit ball and supported in the ball of radius $\sqrt{d}$. Then

$$
\begin{gathered}
(\mu \times \mu \times \cdots \times \mu)\left\{\left(x^{1}, x^{2}, \ldots, x^{d}\right): t \leq \operatorname{det}\left(x^{1}, x^{2}, \ldots, x^{d}\right) \leq t+\epsilon\right\} \\
\approx \iint \ldots \int \psi\left(\frac{\operatorname{det}\left(x^{1}, \ldots, x^{d}\right)-t}{\epsilon}\right) d \mu\left(x^{1}\right) \ldots d \mu\left(x^{d}\right) \\
=\iint \ldots \iint e^{2 \pi i \frac{\lambda}{\epsilon} \operatorname{det}\left(x^{1}, \ldots, x^{d}\right)} e^{-2 \pi i \frac{t \lambda}{\epsilon}} d \mu\left(x^{1}\right) \ldots d \mu\left(x^{d}\right) \widehat{\psi}(\lambda) d \lambda .
\end{gathered}
$$

Using (1.2), it follows that (2.1) equals

$$
\begin{gathered}
\epsilon \int \ldots \int \widehat{\mu}\left(\lambda\left(x^{1} \wedge \cdots \wedge x^{d-1}\right)\right) e^{-2 \pi i t \lambda} d \mu\left(x^{1}\right) \ldots d \mu\left(x^{d-1}\right) \widehat{\psi}(\epsilon \lambda) d \lambda \\
=\epsilon \int \ldots \int_{\left|x^{1} \wedge \cdots \wedge x^{d-1}\right| \leq \lambda^{-1}} \widehat{\mu}\left(\lambda\left(x^{1} \wedge \cdots \wedge x^{d-1}\right)\right) e^{-2 \pi i t \lambda} d \mu\left(x^{1}\right) \ldots d \mu\left(x^{d-1}\right) \widehat{\psi}(\epsilon \lambda) d \lambda \\
+\epsilon \int \ldots \int_{\left|x^{1} \wedge \cdots \wedge x^{d-1}\right|>\lambda^{-1}} \widehat{\mu}\left(\lambda\left(x^{1} \wedge \cdots \wedge x^{d-1}\right)\right) e^{-2 \pi i t \lambda} d \mu\left(x^{1}\right) \ldots d \mu\left(x^{d-1}\right) \widehat{\psi}(\epsilon \lambda) d \lambda:=I+I I .
\end{gathered}
$$

Since $|\widehat{\mu}(\xi)| \leq 1$, we have

$$
I \lesssim \epsilon \int(\mu \times \cdots \times \mu)\left\{\left(x^{1}, \ldots, x^{d-1}\right):\left|x^{1} \wedge \cdots \wedge x^{d-1}\right| \leq \lambda^{-1}\right\}|\widehat{\psi}(\epsilon \lambda)| d \lambda .
$$

Lemma 2.1. With the notation above,

$$
(\mu \times \cdots \times \mu)\left\{\left(x^{1}, \ldots, x^{d-1}\right):\left|x^{1} \wedge \cdots \wedge x^{d-1}\right| \leq \lambda^{-1}\right\} \lesssim \min \left\{1, \lambda^{d-2-s}\right\} .
$$

To prove the lemma, start by noting that one has

$$
\left|x^{1} \wedge x^{2} \wedge \cdots \wedge x^{d-1}\right|=\left|x^{1}\right| \cdot\left|x^{2} \wedge \cdots \wedge x^{d-1}\right| \cdot \sin (\theta),
$$

where $\theta$ is the angle between $x^{1}$ and the $(d-2)$-plane spanned $\Pi$ by $x^{2}, \ldots, x^{d-1}$. Localize to where $\left|x^{2} \wedge \cdots \wedge x^{d-1}\right| \approx 2^{-j}$. By induction, the measure of this set is $\lesssim 2^{j(d-3-s)}$. Since $x^{1}$ is contained in a $2^{j} \lambda^{-1}$-tubular nhood of $\Pi$, it follows that

$$
\begin{aligned}
(\mu \times \cdots \times \mu)\left\{\left(x^{1}, \ldots, x^{d-1}\right):\left|x^{1} \wedge \cdots \wedge x^{d-1}\right| \leq \lambda^{-1}\right\} & \lesssim\left(2^{j} \lambda^{-1}\right)^{-(d-2)}\left(2^{j} \lambda^{-1}\right)^{s}\left(2^{-j}\right)^{s-(d-3)} \\
& =\lambda^{d-2-s} 2^{-j} .
\end{aligned}
$$


It follows that the left hand side of (2.2) is bounded by a constant multiple of

$$
\sum_{j} \lambda^{d-2-s} 2^{-j} \lesssim \lambda^{d-2-s}
$$

completing the proof of Lemma 2.1.

It follows from Lemma 2.1 that

$$
I \lesssim \int \min \left\{1, \lambda^{d-2-s}\right\}|\widehat{\psi}(\epsilon \lambda)| d \lambda,
$$

which is $\lesssim 1$ if $s>d-1$. By the Salem property (1.4),

$$
\begin{gathered}
I I \lesssim \epsilon \int \ldots \int \lambda^{-\frac{s}{2}}\left|x^{1} \wedge \cdots \wedge x^{d-1}\right|^{-\frac{s}{2}} d \mu\left(x^{1}\right) d \mu\left(x^{2}\right) \ldots d \mu\left(x^{d-1}\right)|\widehat{\psi}(\epsilon \lambda)| d \lambda \\
\approx \sum_{j \leq \log _{2}(\lambda)} 2^{\frac{j s}{2}} \epsilon \int \lambda^{-\frac{s}{2}} \mu \times \cdots \times \mu\left\{\left(x^{1}, \ldots, x^{d-1}\right):\left|x^{1} \wedge \cdots \wedge x^{d-1}\right| \approx 2^{-j}\right\}|\widehat{\psi}(\epsilon \lambda)| d \lambda .
\end{gathered}
$$

Using Lemma 2.1, one sees that this is this is

$$
\approx \int \sum_{j \leq \log _{2}(\lambda)} 2^{j(d-2-s)} 2^{\frac{j s}{2}} \lambda^{-\frac{s}{2}}|\widehat{\psi}(\epsilon \lambda)| d \lambda \lesssim \int_{1}^{\infty} \lambda^{d-2-s}|\widehat{\psi}(\epsilon \lambda)| d \lambda
$$

which is bounded independently of $\epsilon$ if $s>d-1$, as desired. This finishes the proof of Thm. 1.6.

\section{Proof of Theorem 1.5}

We shall need the following consequence of Theorem 1.0.3 in [6].

Theorem 3.1. [6] Let $A_{1}, A_{2}, A_{3}, A_{4} \subset[0,1]$, each with $\operatorname{dim}_{\mathcal{H}}\left(A_{j}\right)>\frac{2}{3}$. Then

$$
\mathcal{L}^{1}\left(\left\{a_{1} a_{2}+a_{3} a_{4}: a_{j} \in A_{j}\right\}\right)>0 \text {. }
$$

To apply Thm. 3.1, consider

$$
\operatorname{det}(x, y, z)=y_{1}\left(x_{3} z_{2}-x_{2} z_{3}\right)+y_{2}\left(x_{1} z_{3}-x_{3} z_{1}\right)+y_{3}\left(-x_{1} z_{2}-x_{2} z_{1}\right) .
$$

Fix $x_{1}, x_{2}, x_{3}, y_{3}, z_{3} \in A$, all $\neq 0$, and let

$$
w_{1}=x_{3} z_{2}-x_{2} z_{3}, w_{2}=x_{1} z_{3}-x_{3} z_{1} .
$$

Observe that

$$
-x_{1} z_{2}-x_{2} z_{1}=-\frac{x_{1}}{x_{3}} w_{1}-\frac{x_{2}}{x_{3}} w_{2} .
$$

It follows that the expression in (3.2) equals

$$
y_{1} w_{1}+y_{2} w_{2}-\frac{x_{1}}{x_{3}} w_{1}-\frac{x_{2}}{x_{3}} w_{2}=w_{1}\left(y_{1}-\frac{x_{1}}{x_{3}}\right)+w_{2}\left(y_{2}-\frac{x_{2}}{x_{3}}\right) .
$$

Let

$$
A_{1}=x_{3} B_{2}-x_{2} B_{3}, A_{2}=x_{1} B_{3}-x_{3} B_{1}, A_{3}=B_{1}-\frac{x_{1}}{x_{3}}, A_{4}=B_{2}-\frac{x_{2}}{x_{3}} .
$$


From the assumption that $\operatorname{dim}_{\mathcal{H}}(A)>\frac{2}{3}$, it follows that each $\operatorname{dim}_{\mathcal{H}}\left(A_{j}\right)$ is also $>\frac{2}{3}$. By the above calculation, one has

$$
\left\{a_{1} a_{2}+a_{3} a_{4}: a_{j} \in A_{j}\right\} \subset \mathcal{V}\left(B_{1} \times B_{2} \times B_{3}\right),
$$

and so the conclusion of Theorem 1.5 follows by Theorem 3.1.

\section{Proof of Theorem 1.3}

As noted in the introduction, in view of Thm. 1.1, it suffices to prove Thm. 1.3. For clarity and possible future use, we begin the analysis in $\mathbb{R}^{d}$, specializing to $d=3$ later on. To this end, define a natural measure on the set of wedge products by the relation

$$
\int f(z) d \Lambda(z)=\int \ldots \int f\left(x^{1} \wedge \cdots \wedge x^{d-1}\right) d \mu\left(x^{1}\right) \ldots d \mu\left(x^{d-1}\right) .
$$

It follows that

$$
\widehat{\Lambda}(\xi)=\int \ldots \int e^{-2 \pi i \xi \cdot *\left(x^{1} \wedge \cdots \wedge x^{d-1}\right)} d \mu\left(x^{1}\right) \ldots d \mu\left(x^{d-1}\right)
$$

and thus

$$
\begin{gathered}
\int|\widehat{\Lambda}(\xi)|^{2} \psi\left(\frac{\xi}{R}\right) d \xi \\
=R^{d} \iint \widehat{\psi}\left(R\left(x^{1} \wedge \cdots \wedge x^{d-1}-y^{1} \wedge \cdots \wedge y^{d-1}\right)\right) d \mu\left(x^{1}\right) \ldots d \mu\left(x^{d-1}\right) d \mu\left(y^{1}\right) \ldots d \mu\left(y^{d-1}\right) .
\end{gathered}
$$

Since $\widehat{\psi}$ is rapidly decaying, it suffices to estimate

$$
(\mu \times \cdots \times \mu)\left\{\left(x^{1}, \ldots, x^{d-1}, y^{1}, \ldots, y^{d-1}\right):\left|x^{1} \wedge \cdots \wedge x^{d-1}-y^{1} \wedge \cdots \wedge y^{d-1}\right| \leq R^{-1}\right\} .
$$

Let $B_{r}^{d}(x)$ be the $d$-dimensional ball of radius $r$ centered at $x$; if $x=0$, the center is suppressed. $A_{r}^{d}$ will denote a $d$-dimensional annulus of inner/outer radii $\frac{r}{2}$ and $r$, not necessarily centered at the origin. For both, $d$ is the ambient dimension if not included in the notation; lower dimensional balls and annuli in $\mathbb{R}^{d}$ are denoted using the superscript. Denote $(d-1)$-tuples of vectors in $\mathbb{R}^{d}$ by $\vec{x}=\left(x^{1}, \ldots, x^{d-1}\right)$, and then, as above, $\hat{x}=*\left(x^{1} \wedge \cdots \wedge x^{d-1}\right)$, viewed as a vector.

Let $\mu$ be a Frostman measure on the $s$-dimensional $E \subset A_{1}^{d}$ [14]. To control (4.1), we want to estimate $R^{d} \cdot \mu^{2 d-2}\left(F^{R}\right)$, where

$$
F^{R}:=\{(\vec{x}, \vec{y}):|\hat{x}-\hat{y}|<1 / R\} .
$$

Start by decomposing

$$
B_{1}^{d}=B_{1 / R}(0) \cup \bigcup_{i=0}^{\log _{2} R} \bigcup_{j=0}^{\left(R / 2^{i}\right)^{d}} B_{1 / R}\left(z_{j}^{(i)}\right),
$$


where $\left\{z_{j}^{(i)}\right\}_{j}$ is a $(1 / R)$-net of points in the dyadic shell $A_{2^{-i-1}, 2^{-i}}$. Then we can write

$$
\begin{aligned}
F^{R} & =\left\{(\vec{x}, \vec{y}): \hat{x}, \hat{y} \in B_{1 / R}\right\} \cup \bigcup_{i}\left\{(\vec{x}, \vec{y}): \hat{x}, \hat{y} \in B_{1 / R}\left(z_{j}^{(i)}\right)\right\} \\
& \subset\left(G_{0} \times G_{0}\right) \cup \bigcup_{i} G_{j}^{(i)} \times G_{j}^{(i)},
\end{aligned}
$$

where

$$
G_{0}=\left\{\vec{x}: \hat{x} \in B_{1 / R}\right\}, \quad G_{j}^{(i)}=\left\{\vec{x}: \hat{x} \in B_{1 / R}\left(z_{j}^{(i)}\right)\right\} ;
$$

in terms of measure, the $G_{j}^{(i)}$ essentially only depend on $i$ and we refer to them generically as $G^{(i)}$. With all this, one has

$$
R^{d} \mu^{2 d-2}\left(F^{R}\right) \lesssim R^{d}\left(\mu^{d-1}\left(G_{0}\right)^{2}+\sum_{i=0}^{\log _{2} R}\left(\frac{R}{2^{i}}\right)^{d} \cdot\left(\mu^{d-1}\left(G^{(i)}\right)\right)^{2}\right),
$$

where $\mu^{k}:=\mu \times \cdots \times \mu$, and and we want to estimate the terms on the right hand side.

We now restrict ourselves to three dimensions $(d=3)$.

To estimate the $G_{0}$ term, note that $x^{1} \in E$ is arbitrary, contributing $\mu \lesssim C$. For $x^{1}$ fixed, $x^{2} \in$ a $(1 / R) \times(1 / R) \times 1$ tube, which we cover with $R(1 / R)$-balls, giving a $\mu$ contribution $\lesssim R \cdot R^{-s}=R^{1-s}$. Thus, $\mu^{2}\left(G_{0}\right) \lesssim R^{1-s}$ and hence $\mu^{4}\left(G_{0} \times G_{0}\right) \lesssim R^{2-2 s}$.

To estimate the $G^{(i)}$ term, start by noting that, with $z_{0}:=z_{j}^{(i)}$ fixed, $x^{1}$ must be in a thin nhood of thickness $2^{i} / R$ in $\mathbb{R}^{3}$ of an annulus $A_{1}^{2} \subset z_{0}^{\perp}$; call such a set a washer. Covering this with $\left(R / 2^{i}\right)^{2}$ balls of radius $2^{i} / R$ gives a $\mu \lesssim\left(R / 2^{i}\right)^{2-s}$. For each such $x^{1}$ fixed, $x^{2}$ must satisfy two constraints: (i) It has to be in the same washer as $x^{1}$; and (2) It has to make an angle of $\sim 2^{-i}$ with $x^{1}$ and vary in the radial direction by $\leq 2^{i} / R$. There are thus two cases,

$$
\text { (a) } 1 \leq 2^{i} \leq R^{\frac{1}{2}} ; \text { and }(b) R^{\frac{1}{2}} \leq 2^{i} \leq R \text {. }
$$

In case (a), $2^{i} / R \leq 2^{-i}$, so $x^{2}$ is confined to a $2^{-i} \times\left(2^{i} / R\right) \times\left(2^{i} / R\right)$-tube, which is covered by $R / 2^{2 i}\left(2^{i} / R\right)$-balls, giving

$$
\mu \lesssim\left(R / 2^{2 i}\right)\left(2^{i} / R\right)^{s}=2^{(s-2) i} R^{1-s} .
$$

Multiplying by the $\mu$ measure in $x^{1}$ gives

$$
\mu^{2}\left(G^{(i)}\right) \lesssim 2^{(2 s-4) i} R^{3-2 s} ;
$$

squaring this and multiplying by the prefactor $\left(R / 2^{i}\right)^{d}=R^{3} 2^{-3 i}$ gives

$$
2^{(4 s-11) i} R^{9-4 s}, \quad 1 \leq 2^{i} \leq R^{\frac{1}{2}}
$$

which, since $4 s-11<0$ for the $s$ of interest, takes its largest value for $2^{i}=1$, corresponding to generic configurations and yielding the estimate $R^{9-4 s}$ (before inclusion of the pre-prefactor of $R^{d}=R^{3}$ in $(4.2)$.

In case (b), $2^{i} / R \geq 2^{-i}$ and so $x^{2}$ is confined to a $2^{-i} \times 2^{-i} \times\left(2^{i} / R\right)$-tube, which is covered by $2^{2 i} / R\left(2^{-i}\right)$-balls, giving

$$
\mu \leq\left(2^{2 i} / R\right) \cdot 2^{-s i}=2^{(2-s) i} R^{-1} .
$$

Multiplying by the $\mu$ measure in $x^{1}$, squaring and multiplying by the $R^{3} 2^{-3 i}$ gives $2^{-3 i} R^{5-2 s}$ in the range $R^{\frac{1}{2}} \leq 2^{i} \leq R$. The largest value is for $2^{i}=R^{\frac{1}{2}}$, namely $R^{\frac{7}{2}-2 s}$, but this is smaller than the $R^{9-4 s}$, as is the $G_{0}$ term. 
Thus, we estimate (4.2) by $R^{3} \cdot R^{9-4 s}=R^{12-4 s}$. It follows that the expression in (4.1) is $\lesssim R^{3-(4 s-9)}$, which implies that the Hausdorff dimension of the set $\Lambda(E)$ is greater than or equal to $4 s-9$. To make use of Thm. 1.1, we need $s+(4 s-9)>4$, which holds if $s>\frac{13}{5}$. This completes the proof of Thm. 1.3 and thus of Thm. 1.2.

\section{Applications to discrete geometry}

Definition 5.1. [12] Let $P$ be a set of $n$ points contained in $[0,1]^{2}$. Define the measure

$$
d \mu_{P}^{s}(x)=n^{-1} \cdot n^{\frac{d}{s}} \cdot \sum_{p \in P} \chi_{B^{p}{ }^{p} \frac{1}{s}}(x) d x,
$$

where $\chi_{B_{n}{ }^{p}}(x)$ is the characteristic function of the ball of radius $n^{-\frac{1}{s}}$ centered at $p$. One says that $P$ is $s$-adaptable if

$$
I_{s}\left(\mu_{P}\right)=\iint|x-y|^{-s} d \mu_{P}^{s}(x) d \mu_{P}^{s}(y)<\infty .
$$

This is equivalent to the statement

$$
n^{-2} \sum_{p \neq p^{\prime} \in P}\left|p-p^{\prime}\right|^{-s} \lesssim 1
$$

To understand this condition in clearer geometric terms, suppose that $P$ comes from a 1-separated set $A$, scaled down by its diameter. Then the condition (5.2) takes the form

$$
n^{-2} \sum_{a \neq a^{\prime} \in A}\left|a-a^{\prime}\right|^{-s} \lesssim(\operatorname{diameter}(A))^{-s} .
$$

Thus, $P$ is $s$-adaptable if it is a scaled 1-separated set where the expected value of the distance between two points raised to the power $-s$ is comparable to the value of the diameter raised to the power of $-s$. This basically means that clustering within $P$ is not allowed to be too severe.

In more technical terms, $s$-adaptability means that a discrete point set $P$ can be thickened into a set which is uniformly $s$-dimensional in the sense that its energy integral of order $s$ is finite. Unfortunately, it is shown in [12] that there exist finite point sets which are not s-adaptable for certain ranges of the parameter $s$. The point is that the notion of Hausdorff dimension is much more subtle than the simple "size" estimate. However, many natural classes of sets are $s$-adaptable. For example, homogeneous sets studied by Solymosi and Vu [18] and others are s-adaptable for all $0<s<d$. See also [10] where $s$-adaptability of homogeneous sets is used to extract discrete incidence theorems from Fourier-type bounds.

The following argument is a variant of the conversion mechanism developed in [11, 9]. Suppose that one knew that $\mathcal{L}^{1}(\mathcal{V}(E))>0$ whenever $\operatorname{dim}_{\mathcal{H}}(E)>s_{\mathcal{V}}$. Let $P$ be an $s$-adaptable set with $s \in\left(s_{0}, d\right]$, and $E$ denote the support of $d \mu_{P}^{s}$ above. It follows that

$$
1 \lesssim \mathcal{L}^{1}(\mathcal{V}(E)) \lesssim n^{-\frac{1}{s}} \cdot \# \mathcal{V}(P)
$$


and one can conclude that

$$
\# \mathcal{V}(E) \gtrsim n^{\frac{1}{s}} \gtrsim n^{\frac{1}{s_{0}}}
$$

which yields the following theorem.

Theorem 5.2. Let $P$ be a $\frac{13}{5}$-adaptable subset of $\mathbb{R}^{d}$ of size $n$. Let $\mathcal{V}_{\delta}(P)$ denote the number of distinct $\delta$-separated volumes determined by $P$. Then

$$
\# \mathcal{V}_{n^{-\frac{5}{13}}}(P) \gtrsim n^{\frac{5}{13}}
$$

It is important to note that the significance of this result lies in the requirement that the volumes are $n^{-\frac{5}{13}}$-separated. In the absence of this feature, the exponent is inferior to the sharp result obtained by Dumitrescu and Toth [3], who proved that for any points set $P \subset \mathbb{R}^{3}$ of size $n$, $\# \mathcal{V}(P) \gtrsim n$.

Even if one were to improve Thm. 1.2 to the conjecturally sharp $\operatorname{dim}_{\mathcal{H}}(E)>2$, the resulting modification to Thm. 5.2 would be that $\# \mathcal{V}_{n^{-\frac{1}{2}}}(P) \gtrsim n^{\frac{1}{2}}$, still weaker in terms of the exponent than [3]. The analytic method used here yields a conclusion about $n^{-\frac{1}{s}}$-separated volumes; we do not know whether it is possible to obtain the sharp exponent using our methods.

\section{References}

[1] P. Brass, W. Moser and J Pach, Research Problems in Discrete Geometry, Springer (2000). 1

[2] D. Covert, D. Hart, A. Iosevich, D. Koh and M. Rudnev, Generalized incidence theorems, homogeneous forms and sum-product estimates in finite fields, European J. of Combinatorics 31, 2010, 306-319. 2

[3] A. Dumitrescu and C. Toth, On the number of tetrahedra with minimum, unit, and distinct volumes in threespace. (preprint), (2011). 8

[4] B. Erdog̃an A bilinear Fourier extension theorem and applications to the distance set problem IMRN (2006). 1

[5] B. Erdog̃an, A. Iosevich and K. Taylor Finite point configurations, uniform distribution, intersections of fractals, and number theoretic consequences, (in preparation), (2011). 1

[6] B. Erdog̃an, D. Hart and A. Iosevich, Multi-parameter projection theorems with applications to sums-products and finite point configurations in the Euclidean setting, to appear in the Birkhauser volume in honor of Kostya Oskolkov's 65th birthday, (2011). 2, 4

[7] K. J. Falconer On the Hausdorff dimensions of distance sets, Mathematika 32 (1986), 206-212. 1

[8] K. J. Falconer, The geometry of fractal sets, Cambridge Tracts in Mathematics, 85 Cambridge University Press, Cambridge, (1986). 2

[9] S. Hofmann and A. Iosevich, A Circular averages and Falconer/Erds distance conjecture in the plane for random metrics, Proc. Amer. Math. Soc. 133 (2005), no. 1, 133-143. 7

[10] A. Iosevich, H. Jorati and I. Laba, Geometric incidence theorems via Fourier analysis, Trans. Amer. Math. Soc. 361 (2009), no. 12, 6595-6611. 7

[11] A. Iosevich, I. Laba, K-distance sets, Falconer conjecture, and discrete analogs, Integers 5 (2005), no. 2. 7

[12] A. Iosevich, M. Rudnev and I. Uriarte-Tuero, Theory of dimension for large discrete sets and applications, (arXiv:0707.1322). 7

[13] J. Matousek, Lectures on Discrete Geometry, Graduate Texts in Mathematics, Springer 202 (2002). 1

[14] P. Mattila, Geometry of sets and measures in Euclidean spaces, Cambridge University Press, volume 44, (1995). 2,5

[15] J. Pach Directions in combinatorial geometry, Jahresber. Deutsch. Math.-Verein. 107 (2005), no. 4, 215-225. 1

[16] J. Pach and M. Sharir Geometric incidences, Towards a theory of geometric graphs, 185-223, Contemp. Math., 342, Amer. Math. Soc., Providence, RI, (2004). 1

[17] L. Szekely, A. Crossing numbers and hard Erdős problems in discrete geometry Combin. Probab. Comput. 6 (1997), 353-358. 1

[18] J. Solymosi and V. Vu, Distinct distances in high dimensional homogeneous sets, Towards a theory of geometric graphs, 259-268, Contemp. Math., 342, Amer. Math. Soc., Providence, RI, (2004). 7

[19] T. Wolff, Decay of circular means of Fourier transforms of measures, International Mathematics Research Notices 10 (1999) 547-567. 1 
Department of Mathematics, University of Rochester, Rochester, NY 14627

E-mail address: allan@math.rochester.edu

Department of Mathematics, University of Rochester, Rochester, NY 14627

E-mail address: iosevich@math.rochester.edu

Department of Mathematics, Université Paris-Sud 11, Orsay

E-mail address: mihalis.mourgoglou@math.u-psud.fr 